\title{
Modelling the impact of plasma therapy and immunotherapy for recovery of COVID-19 infected individuals
}

\author{
Nita H. Shah ${ }^{1}$ - Ankush H. Suthar ${ }^{1}$ Ekta N. Jayswal ${ }^{1} \cdot$ Nehal Shukla $^{2}$. \\ Jagdish Shukla ${ }^{3}$
}

Accepted: 27 March 2021 / Published online: 12 April 2021

(c) Instituto de Matemática e Estatística da Universidade de São Paulo 2021

\begin{abstract}
Since the first case of COVID-19 was detected in Wuhan, China in December 2019, COVID-19 has become a pandemic causing a global economic and public health emergency. There is no known treatment or vaccine available for COVID-19 during the initial period of the outbreak. Immunotherapy and plasma therapy has been used with satisfactory efficacy over the past two decades in many viral infections like SARS (Systemic Acute Respiratory Syndrome), MERS (Middle East Respiratory Syndrome) and H1N1. Limited data from China show clinical benefit, radiological resolution, reduction in viral loads and improved survival. We aim to create a mathematical model for COVID-19 transmission and then apply various control parameters to see their effects on recovery from COVID-19 disease. We have formulated a system of non-linear ordinary differential equations, calculated basic reproduction $R_{0}$ and applied five different controls (self-isolation, quarantine, herd immunity, immunotherapy, plasma therapy) to test the effectiveness of plasma therapy. Control optimality was checked by Lagrangian functions. Numerical simulations and bifurcation analysis were carried out. The study concludes that the COVID-19 outbreak can be controlled up to a significant level in three weeks after applying all the control strategies together. These strategies lead to reduction in hospitalization and a rise in recovery from infection. Immunotherapy is highly effective initially in hospitalized infected individuals however better results were seen in the long term with plasma therapy.
\end{abstract}

Keywords Modelling · COVID-19 · Immunotherapy · Convalescent Plasma • Control Strategy

Communicated by José Alberto Cuminato.

Nehal Shukla

shukla_nehal@columbusstate.edu

Extended author information available on the last page of the article 


\section{Introduction}

The first case of unknown pneumonia was detected in Wuhan China in December 2019, and was later identified as COVID-19 spread by SARS COV-2 (severe acute respiratory syndrome coronavirus 2) (Cohen and Normile 2020). COVID19 was declared a pandemic on $11^{\text {th }}$ March by WHO. It has spread to 210 countries and as per worldometer (Worldometer 2020) and as of 28th February 2021 worldwide total cases are around 114,379,825 and total deaths are 2,537,578. It caused global economic and public health emergencies. It spreads by direct or indirect contact with respiratory droplets from infected individuals (Holshue et al. 2020). Several steps like physical distancing, quarantine and other sanitizing habits have shown some success in slowing down the pandemic but it is still far from being contained in most countries.

There is no known treatment or vaccine available in the initial period of the outbreak. The antimalarial drug chloroquine and antibiotic azithromycin have shown some hope against COVID-19 but its efficacy has been recently debated (Syal 2020). Other potential therapeutic agents tried so far include Remdesivir, Lopinavir / Ritonavir (Kaletra), Toclizumab (Actemra), Remdesivir have shown some promise in controlling the COVID-19 disease. (Holshue et al. 2020; Lu 2020; Russell et al. 2020; Wang et al. 2020). Most people in developing countries cannot afford costly therapeutic interventions like mechanical ventilators and prolonged lockdown, pandemic must be curtailed so that health infrastructure can manage it efficiently. In absence of known treatment and proper vaccine, herd immunity can help in decreasing its spread. (Syal 2020).

Immunotherapy and plasma therapy has been used effectively as a therapeutic option against many viral infections. The main methods in immunotherapy include several vaccines and monoclonal antibody candidates. Convalescent plasma (CP) therapy has been used for the prevention and treatment of many infectious diseases for more than one century. In both SARS-CoV (Severe Acute Respiratory Syndrome Corona Virus), and SARS-CoV-2 viruses' entry into the host cells is mediated by interaction of the receptor-binding domain (RBD) in $S$ protein on virus outer-membrane and angiotensin-converting enzyme 2 (ACE2) on cell. So, these proteins can be the major potential targets for immunotherapy (AminJafari and Ghasemi 2020; Duan et al. 2020b). CP refers to plasma that is collected from individuals, following resolution of infection and development of antibodies. Over the past two decades, CP therapy was successfully used in the treatment of SARS MERS (Middle East Respiratory Syndrome), and 2009 H1N1 pandemic with satisfactory efficacy and safety (Cheng et al. 2005; Zhou et al. 2007; Hung et al. 2011; Ko et al. 2018).

A meta-analysis from 32 studies of SARS coronavirus infection and severe influenza showed a statistically significant reduction in the pooled odds of mortality following CP therapy, compared with placebo or no therapy (odds ratio, 0.25; 95\% confidence interval, 0.14-0.45) (Mair-Jenkins et al. 2015). Since SARS, MERS, and COVID-19 share similar virologic and clinical symptomatology (Lee and Hsueh 2020), CP therapy might be a promising treatment option 
for COVID-19 treatment (Chen et al. 2020). Studies were done by Cheng et al in 2005,(Cheng et al. 2005) in Hong Kong reported that in 2003 during SARS outbreak, patients who received convalescent plasma had a lower mortality rate (12.5\%) compared with the overall SARS-related mortality for admitted patients $(\mathrm{n}=299[17 \%])$.

Convalescent plasma (CP) has also been used in the COVID-19 pandemic; limited data from China suggest clinical benefit, radiological resolution, reduction in viral loads and improved survival (Bloch et al. 2020). While fractionated plasma products (e.g. hyperimmune globulin, monoclonal antibodies) and/or vaccination may offer durable therapeutic options, human anti-SARS-CoV-2 plasma is the only therapeutic option that is immediately available for use to treat COVID-19 (Bloch et al. 2020). A study was done by Shen et al, (2020) and Duan et al. (2020b) concluded that convalescent plasma therapy is well tolerated and could potentially improve the clinical outcome. They collected CP from patients three weeks after they recovered from COVID-19, and from the patients who were having normal body temperature for $>3$ days, not having respiratory symptoms and their two consecutive PCR SARS COV-2 test 24 hours apart has been negative. Their study result showed a decline in inflammatory markers, improvement in the patient's antibody titre and PCR SARS COV-2 became negative. CFR (Case Fatality Rate) was 0 in the study done by Duan et al., however, there were several limitations of the study including small sample size, lack of randomized double blind controlled study, those patients received another medication like antivirals (kaletra), steroids at the same time, and it is difficult to attribute all improvement to plasma therapy alone. Their study indicates that convalescent plasma therapy could be the most critical weapon in the fight with COVID-19 in severe cases. Survivors of the COVID-19 may play a key role in both herd immunity as well as the availability of plasma therapy. (Syal 2020).

Since, the effective vaccine and specific antiviral medicines are unavailable before 2021, people use mathematical modeling for analysis of the COVID-19 transmission and alternative strategy for COVID-19 treatment, especially among severe patients. Ndaïrou et al. (2020) have proposed a new epidemiological compartment model, with a special focus on the transmissibility of super-spreaders individuals. Our aim for the present study is to create a compartmental mathematical model for the spread of COVID-19 with specific transmissibility of active spreaders and super active spreaders of the infection. Moreover, various control parameters are applied in the model like self-isolation, quarantine of infected individuals and hospitalization to receive medication (immunotherapy and convalescent plasma therapy) and see their effects on recovery from COVID-19 disease.

\section{Mathematical model}

In this section, a basic model for COVID-19 transmission dynamics among humans is constructed. Infected individuals who regularly come in contact with exposed class, for example, vegetable vendor, grocery store-keeper, policeman or security man, delivery man, etc., all unknowingly spread infection at a high rate. Hence, 
these population classes are considered to be super active spreaders of the infection. Remaining all infected (symptomatically and asymptomatically) individuals who are capable to infect others are considered to be active spreaders of COVID-19 infection. The model contains these two different classes of infected who are the spreader of the infection and accelerate the intensity of the COVID-19 outbreak. In some cases, the strong immunity of an infected individual can defeat the infection, making the individual infection free without hospitalisation. Moreover, there are particular cases in which recovered individuals present the symptoms of the disease and hence they need to get hospitalisation again. These two situations are also considered in the present model.

In the proposed model, the total population is divided into seven compartments: exposed (not infectious) individuals $(E)$, infected individuals $(I)$, infected individuals who are active spreaders $(A)$, infected individuals who are super active spreaders $(S)$, quarantined individuals $(Q)$, hospitalised individuals $(H)$ and recovered individuals $(R)$. Using Fig. 1, the model is formulated as dynamics of non-linear differential equation as given below.

$$
\begin{aligned}
& E^{\prime}=B-\beta_{1} E I \\
& I^{\prime}=\beta_{1} E I-\beta_{2} I A-\beta_{3} I S \\
& A^{\prime}=\beta_{2} I A-\beta_{4} A+\beta_{5} S-\beta_{6} A-\beta_{7} A \\
& S^{\prime}=\beta_{3} I S+\beta_{4} A-\beta_{5} S-\beta_{8} S-\beta_{9} S \\
& Q^{\prime}=\beta_{6} A+\beta_{8} S-\beta_{10} Q-\beta_{13} Q \\
& H^{\prime}=\beta_{7} A+\beta_{9} S-\beta_{11} H+\beta_{12} R+\beta_{13} Q-\mu_{d} H \\
& R^{\prime}=\beta_{10} Q+\beta_{11} H-\beta_{12} R
\end{aligned}
$$

Here $\beta_{2}$ and $\beta_{3}$ are ratio of active spreader and super active spreader respectively to the total infected individuals. $\beta_{4}$ is the ratio of number of individuals who become super-active spreader from active spreader to the total number of active spreaders. Similarly, $\beta_{5}$ is the ratio of number of super-active spreaders who become active spreaders to the total number of super-active spreaders. Rate $\beta_{6}$ is the ratio of quarantined active spreaders to the total number of active spreaders and $\beta_{8}$ is the ratio of quarantined

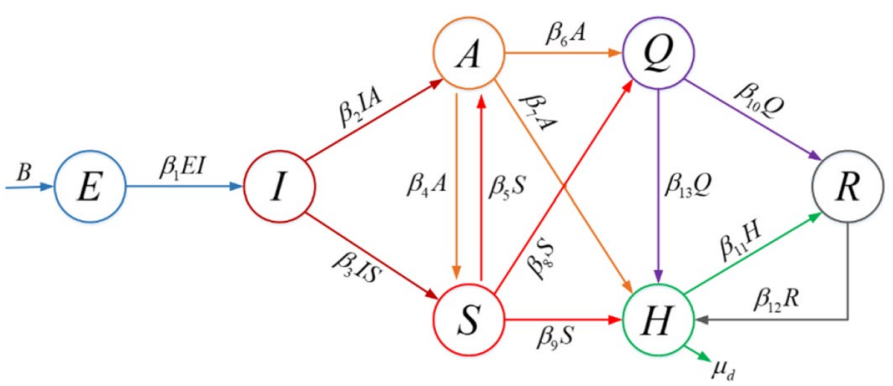

Fig. 1 System diagram of COVID-19 transmission model 
super-active spreaders to the total number of super-active spreaders. Similarly, $\beta_{7}$ and $\beta_{9}$ are the hospitalisation rates of active spreaders and super-active spreaders. Recovery rates $\beta_{10}$ and $\beta_{11}$ are the ratio of number of recovered from the respective compartment to the number of quarantined and hospitalised individuals respectively. And $\beta_{12}$ is the ratio of hospitalised individuals after recovered from COVID-19 infection to the total recovered individuals. Parameters used in the model are listed in the Table 1.

\subsection{Well-posedness of the solution}

In this sub-section, we observe that the solutions of the system (1) are non-negative and bounded if initial conditions are non-negative (Mahrouf, M. et al., 2021). Since the model contains only human population, only non-negative initial conditions are used. Also, all the parameters used in the model are considered non-negative. We know that $(E(t), I(t), A(t), S(t), Q(t), H(t), R(t)) \geq 0$ if $(E(0), I(0), A(0), S(0), Q(0), H(0), R(0)) \geq 0$. Based on system (1), we have $N=B-\mu_{d} H$. Where $N=E+I+A+S+Q+H+R$. When $t \rightarrow \infty$, we have $N \leq B$, since $\mu_{d} \geq 0$. Hence $N$ is bounded and the feasible region is $\Lambda$ :

$$
\Lambda=\left\{(E, I, A, S, Q, H, R) \in R_{+}^{7}: E+I+A+S+Q+H+R \leq B\right\}
$$

Table 1 Parameters used in the model. Note that, data for total number of infected cases, critical cases, hospitalised cases and deaths due to COVID-19 are taken from worldometers on 25th April, 2020, (Worldometer 2020)

\begin{tabular}{lll}
\hline Parameters & & Parametric value \\
\hline$B$ & Recruitment rate of exposed individuals & 0.60 \\
$\beta_{1}$ & Recruitment rate of infected individuals & 0.17 \\
$\beta_{2}$ & Rate at which infected individuals become active spreader & 0.40 \\
$\beta_{3}$ & Rate at which infected individuals become super active spreader & 0.20 \\
$\beta_{4}$ & Rate at which active spreader become super active spreader & 0.05 \\
$\beta_{5}$ & Rate at which super active spreader become active spreader & 0.30 \\
$\beta_{6}$ & Rate at which active spreader moves to quarantine & 0.02 \\
$\beta_{7}$ & Rate at which active spreader moves to hospitalisation & 0.70 \\
$\beta_{8}$ & Rate at which super active spreader moves to quarantine & 0.30 \\
$\beta_{9}$ & Rate at which super active spreader moves to hospitalisation & 0.70 \\
$\beta_{10}$ & Recovery rate of quarantined individuals without hospitalisation & 0.07 \\
$\beta_{11}$ & Recovery rate of hospitalised individuals & 0.44 \\
$\beta_{12}$ & Rate at which recovered individuals again moves to hospitalisation & 0.30 \\
$\beta_{13}$ & Rate at which quarantined individuals get hospitalisation & 0.60 \\
$\mu_{d}$ & Mortality rate of COVID-19 & 0.1077 \\
\hline
\end{tabular}

Approximate parametric values are calculated using the data available and some are assumed 


\subsection{Basic reproduction number}

The basic reproduction number $R_{0}$ is defined as the average number of secondary infected cases rising from an average primary case in an entirely exposed/susceptible population.

The solution of the system (1), the endemic equilibrium point $E_{p}^{*}=\left(E^{*}, I^{*}, A^{*}, S^{*}, Q^{*}, H^{*}, R^{*}\right)$ is as follow:

$$
\begin{aligned}
& E^{*}=\frac{B}{r \beta_{1}}, I^{*}=r, A^{*}=\frac{B\left(\beta_{3} r-\beta_{5}-\beta_{8}-\beta_{9}\right)}{q}, S^{*}=-\frac{B \beta_{4}}{q} \\
& Q^{*}=\frac{B\left(\left(\beta_{3} r-\beta_{5}\right) \beta_{6}-\beta_{6}\left(\beta_{8}+\beta_{9}\right)-\beta_{4} \beta_{8}\right)}{q\left(\beta_{10}+\beta_{13}\right)}, H^{*}=\frac{B}{\mu_{d}}, R^{*}=\frac{p B}{q\left(\beta_{10}+\beta_{13}\right) \mu_{d} \beta_{12}}
\end{aligned}
$$

where,

$$
\begin{aligned}
p= & \mu_{d} \beta_{10} \beta_{6}\left(\beta_{3} r-\beta_{5}\right)-\mu_{d} \beta_{10} \beta_{4} \beta_{8}+\beta_{11}\left(\beta_{6}+\beta_{7}\right)\left(\beta_{3} r-\beta_{5}\right)\left(\beta_{10}+\beta_{13}\right) \\
& +\left(\beta_{8}+\beta_{9}\right)\left(\beta_{11}\left(\beta_{6}+\beta_{7}\right)\left(\beta_{10}+\beta_{13}\right)+\beta_{11} \beta_{4}\left(\beta_{10}+\beta_{13}\right)+\mu_{d} \beta_{10} \beta_{6}\right)
\end{aligned}
$$

$q=\left(\beta_{6}+\beta_{7}\right)\left(\beta_{3} r-\beta_{5}\right)-\left(\beta_{8}+\beta_{9}\right)\left(\beta_{4}+\beta_{6}+\beta_{7}\right)$ and $r$ is the highest root of a polynomial $m(z)=a_{0} z^{2}+a_{1} z+a_{2}=0$, coefficients of the polynomial are: $a_{0}=\beta_{2} \beta_{3}$, $a_{1}=-\left(\beta_{2}\left(\beta_{5}+\beta_{8}+\beta_{9}\right)+\beta_{3}\left(\beta_{4}+\beta_{6}+\beta_{7}\right)\right)$ and $a_{2}=\beta_{5}\left(\beta_{6}+\beta_{7}\right)+\left(\beta_{8}+\beta_{9}\right)\left(\beta_{4}+\beta_{6}+\beta_{7}\right)$. This $R_{0}$ based on endemic equilibrium point can be calculated using next generation matrix method (Diekmann et al. 1990; Garba et al. 2008). The above system (1) can be written in the following form using matrices.

$$
f=\left[\begin{array}{c}
\beta_{1} E I \\
\beta_{2} I A \\
\beta_{3} I S \\
0 \\
0 \\
0 \\
0
\end{array}\right] \text { and } v=\left[\begin{array}{c}
\beta_{2} I A+\beta_{3} I S \\
\beta_{4} A-\beta_{5} S+\beta_{6} A+\beta_{7} A \\
-\beta_{4} A+\beta_{5} S+\beta_{8} S+\beta_{9} S \\
-B+\beta_{1} E I \\
-\beta_{6} A-\beta_{8} S+\beta_{10} Q+\beta_{13} Q \\
-\beta_{7} A-\beta_{9} S+\beta_{11} H-\beta_{12} R-\beta_{13} Q+\mu_{d} H \\
-\beta_{10} Q-\beta_{11} H+\beta_{12} R
\end{array}\right]
$$

Note that, matrix $F$ shows the new infectious rates and matrix $V$ shows other rates transferred in between the compartments, are given respectively by:

$$
F=\left[\begin{array}{ccccccc}
\beta_{1} E^{*} & 0 & 0 & \beta_{1} I^{*} & 0 & 0 & 0 \\
\beta_{2} A^{*} & \beta_{2} I^{*} & 0 & 0 & 0 & 0 & 0 \\
\beta_{3} S^{*} & 0 & \beta_{3} I^{*} & 0 & 0 & 0 & 0 \\
0 & 0 & 0 & 0 & 0 & 0 & 0 \\
0 & 0 & 0 & 0 & 0 & 0 & 0 \\
0 & 0 & 0 & 0 & 0 & 0 & 0 \\
0 & 0 & 0 & 0 & 0 & 0 & 0
\end{array}\right]
$$




$$
V=\left[\begin{array}{ccccccc}
\beta_{2} A^{*}+\beta_{3} S^{*} & \beta_{2} I^{*} & \beta_{3} I^{*} & 0 & 0 & 0 & 0 \\
0 & \beta_{4}+\beta_{6}+\beta_{7} & -\beta_{5} & 0 & 0 & 0 & 0 \\
0 & -\beta_{4} & \beta_{5}+\beta_{8}+\beta_{9} & 0 & 0 & 0 & 0 \\
\beta_{1} E^{*} & 0 & 0 & \beta_{1} I^{*} & 0 & 0 & 0 \\
0 & -\beta_{6} & -\beta_{8} & 0 & \beta_{10}+\beta_{13} & 0 & 0 \\
0 & -\beta_{7} & -\beta_{9} & 0 & -\beta_{13} & \beta_{11}+\mu_{d} & -\beta_{12} \\
0 & 0 & 0 & 0 & -\beta_{10} & -\beta_{11} & \beta_{12}
\end{array}\right]
$$

Hence, the basic reproduction number $\left(R_{0}\right)$, is given by

$$
\begin{aligned}
R_{0}=\rho\left(F V^{-1}\right)= & \frac{\beta_{2} \beta_{3} r\left(\left(\beta_{6}+\beta_{7}\right)\left(\beta_{3} r-\beta_{5}\right)-\left(\beta_{8}+\beta_{9}\right)\left(\beta_{4}+\beta_{6}+\beta_{7}\right)\right)}{\left(\beta_{6}+\beta_{7}\right)\left(\beta_{2} \beta_{5}\left(\beta_{3} r-\beta_{5}\right)-\beta_{3} \beta_{4} \beta_{5}\right)+\left(\beta_{8}+\beta_{9}\right)\left(\beta_{3} \beta_{4}\left(\beta_{2} r-\beta_{4}\right)-\beta_{2} \beta_{4} \beta_{5}\right.} \\
& \left.-\beta_{2}\left(\beta_{8}+\beta_{9}\right)\left(\beta_{4}+\beta_{6}+\beta_{7}\right)+\left(\beta_{6}+\beta_{7}\right)\left(\beta_{2} \beta_{3} r-2 \beta_{2} \beta_{5}-\beta_{3} \beta_{4}\right)\right)
\end{aligned}
$$

where $\rho$ is the dominant eigenvalue in magnitude of the matrix $F V^{-1}$. After substituting all parametric values from Table 1 , we get the threshold value $R_{0}=3.4709$ which represent the average number of secondary cases generated by an infected individual in completely susceptible population. From this calculation, we can say the model or the current outbreak is in highly unstable (Driessche \& Watmough, 2002), hence, certain control strategies are very essential to impose to come out from pandemic situation.

\section{Optimal control}

In this section, we extend the system (1) to include five time dependent control strategies, $u_{1}(t), u_{2}(t), u_{3}(t), u_{4}(t)$ and $u_{5}(t)$, regarding isolation of infected individuals and medication to improve immunity to fight against the COVID-19 outbreak.

Here, control variables $u_{1}(t)$ and $u_{2}(t)$ measure the quarantine or isolation of the individuals who are spreader and super spreader respectively. We can provide herd immunity or herd protection to those who are not immune to the COVID-19 disease by improving immunity in most of the population. Also strong immunity of large mass can control further spread of COVID-19 infection. The control variable $u_{3}(t)$ indicates the strategy which increases the herd immunity which helps quarantined individuals to get recovered without medication. The control variable $u_{4}(t)$ suggests to provide proper immunotherapy (include several types of vaccines, monoclonal antibody candidates, etc.) to hospitalised individuals which helps them to fight against the viral infection. In present situation, where proper vaccination is not available for the COVID-19, a convalescent plasma therapy have sparked a ray of hope. The Convalescent plasma therapy's effects as treatment for Covid-19 has been tested positive with no severe adverse effects (Duan et al. 2020a, b; Shen et al. 2020). The control variable $u_{5}(t)$ is used to support convalescent plasma therapy to improve immunity of critically infected individuals. However, the limitation of this control strategy is, if plasma therapy is not used properly and carefully, it can create more serious complications. 
Under these assumptions, the COVID-19 model (1) is re-constructed by including control variables from Fig. 2:

$$
\begin{aligned}
& E^{\prime}=B-\beta_{1} E I \\
& I^{\prime}=\beta_{1} E I-\beta_{2} I A-\beta_{3} I S+u_{1} A+u_{2} S \\
& A^{\prime}=\beta_{2} I A-\beta_{4} A+\beta_{5} S-\beta_{6} A-\beta_{7} A-u_{1} A \\
& S^{\prime}=\beta_{3} I S+\beta_{4} A-\beta_{5} S-\beta_{8} S-\beta_{9} S-u_{2} S \\
& Q^{\prime}=\beta_{6} A+\beta_{8} S-\beta_{10} Q-\beta_{13} Q-u_{3} Q \\
& H^{\prime}=\beta_{7} A+\beta_{9} S-\beta_{11} H+\beta_{12} R+\beta_{13} Q-\mu_{d} H-u_{4} H-u_{5} R \\
& R^{\prime}=\beta_{10} Q+\beta_{11} H-\beta_{12} R+u_{4} H+u_{5} R+u_{3} Q
\end{aligned}
$$

According to this extended model, the optimal control problem with the objective function is formulated by

$$
\begin{aligned}
\text { Optimise } J\left(u_{1}, u_{2}, u_{3}, u_{4}, u_{5}\right) & =\int_{0}^{T}\left(A_{1} E(t)+A_{2} I(t)+A_{3} A(t)+A_{4} S(t)+A_{5} Q(t)+A_{6} H(t)+A_{7} R(t)\right. \\
& \left.+w_{1} u_{1}^{2}(t)+w_{2} u_{2}^{2}(t)+w_{3} u_{3}^{2}(t)+w_{4} u_{4}^{2}(t)+w_{5} u_{5}^{2}(t)\right) d t
\end{aligned}
$$

The objective is to minimise active and super active spreaders, increase recovery of hospitalised and quarantined individuals. In equation (9), $A_{i}, i=1,2, \ldots 7$, are weight constants of the state variables and $w_{j}, j=1,2, \ldots, 5$ are weight constants of respective control variables. Our goal is to determine optimal control functions $\left(u_{1}^{*}, u_{2}^{*}, u_{3}^{*}, u_{4}^{*}, u_{5}^{*}\right)$, such that

$$
J\left(u_{1}^{*}, u_{2}^{*}, u_{3}^{*}, u_{4}^{*}, u_{5}^{*}\right)=\operatorname{optimise}\left(J\left(u_{1}, u_{2}, u_{3}, u_{4}, u_{5}\right) /\left(u_{1}, u_{2}, u_{3}, u_{4}, u_{5}\right) \in \phi\right)
$$

subject to the modified system (8), where $\phi$ is a control strategy set. $\phi=\left\{\left(u_{1}, u_{2}, u_{3}, u_{4}, u_{5}\right) / u_{i}(t)\right.$ is Lebesgue measurable on $\left.[0, T], 0 \leq u_{i}(t) \leq 1, i=1,2, \ldots, 5\right\}$

Theorem 1 Consider the objective function (9) with $\left(u_{1}, u_{2}, u_{3}, u_{4}, u_{5}\right) \in \Gamma$ subject to the constraint state system (8) then there exist $\left(u_{1}^{*}, u_{2}^{*}, u_{3}^{*}, u_{4}^{*}, u_{5}^{*}\right) \in \Gamma$ such that $J\left(u_{1}^{*}, u_{2}^{*}, u_{3}^{*}, u_{4}^{*}, u_{5}^{*}\right)=\operatorname{optimise}\left(J\left(u_{1}, u_{2}, u_{3}, u_{4}, u_{5}\right) /\left(u_{1}, u_{2}, u_{3}, u_{4}, u_{5}\right) \in \phi\right)$.

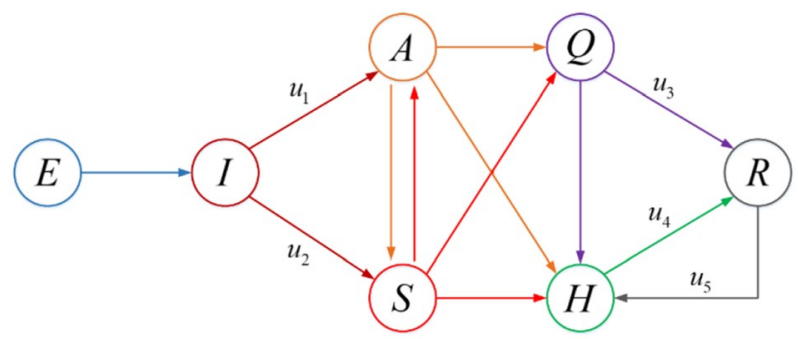

Fig. 2 COVID-19 model with control variables 
Proof The

integrand,

$A_{1} E(t)+A_{2} I(t)+A_{3} A(t)+A_{4} S(t)+A_{5} Q(t)+A_{6} H(t)+A_{7} R(t)+w_{1} u_{1}^{2}(t)$

$+w_{2} u_{2}^{2}(t)+w_{3} u_{3}^{2}(t)+w_{4} u_{4}^{2}(t)+w_{5} u_{5}^{2}(t)$ of the objective function (9) is convex in the set $\phi$. The control strategy set $\phi$ is also close and convex by definition. Since the model (8) is bounded and linear in the control variables, the conditions for the existence of optimal control are satisfied (Fleming and Rishel 1975).

\subsection{Optimality system}

Let us convert the problem into a problem of maximizing a Lagrangian function $L$, with respect to all control variables $u_{1}, u_{2}, u_{3}, u_{4}$ and $u_{5}$. For necessary condition of an optimal control problem, Pontryagins maximum principle (Pontryagin 2018) is used.

$$
\begin{aligned}
L= & A_{1} E^{2}(t)+A_{2} I^{2}(t)+A_{3} A^{2}(t)+A_{4} S^{2}(t)+A_{5} Q^{2}(t)+A_{6} H^{2}(t)+A_{7} R^{2}(t)+w_{1} u_{1}^{2}+w_{2} u_{2}^{2}+w_{3} u_{3}^{2} \\
& +w_{4} u_{4}^{2}+w_{5} u_{5}^{2}+\lambda_{1}\left(B-\beta_{1} E I\right)+\lambda_{2}\left(\beta_{1} E I-\beta_{2} I A-\beta_{3} I S+u_{1} A+u_{2} S\right)+\lambda_{3}\left(\beta_{2} I A-\beta_{4} A+\beta_{5} S\right. \\
& \left.-\beta_{6} A-\beta_{7} A-u_{1} A\right)+\lambda_{4}\left(\beta_{3} I S+\beta_{4} A-\beta_{5} S-\beta_{8} S-\beta_{9} S-u_{2} S\right)+\lambda_{5}\left(\beta_{6} A+\beta_{8} S-\beta_{10} Q-\beta_{13} Q\right. \\
& \left.-u_{3} Q\right)+\lambda_{6}\left(\beta_{7} A+\beta_{9} S-\beta_{11} H+\beta_{12} R+\beta_{13} Q-\mu_{d} H-u_{4} H-u_{5} R\right)+\lambda_{7}\left(\beta_{10} Q+\beta_{11} H-\beta_{12} R\right. \\
& \left.+u_{4} H+u_{5} R+u_{3} Q\right)
\end{aligned}
$$

For given an optimal control $u^{*}=\left(u_{1}^{*}, u_{2}^{*}, u_{3}^{*}, u_{4}^{*}, u_{5}^{*}\right)$ and corresponding state solutions of the system (8), there exist adjoint functions, $\lambda_{i}, i=1,2, \ldots 7$, which are

$$
\begin{gathered}
\lambda_{1}^{\prime}=-\frac{\partial L}{\partial E}=-2 A_{1} E+\beta_{1} I\left(\lambda_{1}-\lambda_{2}\right) \\
\lambda_{2}^{\prime}=-\frac{\partial L}{\partial I}=-2 A_{2} I+\beta_{1} E\left(\lambda_{1}-\lambda_{2}\right)+\beta_{2} A\left(\lambda_{2}-\lambda_{3}\right)+\beta_{3} S\left(\lambda_{2}-\lambda_{3}\right) \\
\lambda_{3}^{\prime}=-\frac{\partial L}{\partial A}=-2 A_{3} A+\left(\beta_{2} I-u_{1}\right)\left(\lambda_{2}-\lambda_{3}\right)+\beta_{4}\left(\lambda_{3}-\lambda_{4}\right)+\beta_{6}\left(\lambda_{3}-\lambda_{5}\right)+\beta_{7}\left(\lambda_{3}-\lambda_{6}\right) \\
\lambda_{4}^{\prime}=-\frac{\partial L}{\partial S}=-2 A_{4} S+\left(\beta_{3} I-u_{2}\right)\left(\lambda_{2}-\lambda_{4}\right)+\beta_{5}\left(\lambda_{4}-\lambda_{3}\right)+\beta_{8}\left(\lambda_{4}-\lambda_{5}\right)+\beta_{9}\left(\lambda_{4}-\lambda_{6}\right) \\
\lambda_{5}^{\prime}=-\frac{\partial L}{\partial Q}=-2 A_{5} Q+\left(\beta_{10}+u_{3}\right)\left(\lambda_{5}-\lambda_{7}\right)+\beta_{13}\left(\lambda_{5}-\lambda_{6}\right) \\
\lambda_{6}^{\prime}=-\frac{\partial L}{\partial H}=-2 A_{6} H+\left(\beta_{11}+u_{4}\right)\left(\lambda_{6}-\lambda_{7}\right)+\mu_{d} \lambda_{6} \\
\lambda_{7}^{\prime}=-\frac{\partial L}{\partial R}=-2 A_{7} R+\left(\beta_{12}-u_{5}\right)\left(\lambda_{7}-\lambda_{6}\right)
\end{gathered}
$$

The terminal conditions are $\lambda_{i}(T)=0$, for $i=1,2, \ldots 7$. The optimal control variables $u_{1}^{*}, u_{2}^{*}, u_{3}^{*}, u_{4}^{*}$ and $u_{5}^{*}$ can be solves using optimality conditions 


$$
\begin{aligned}
& -\frac{\partial L}{\partial u_{i}}=0, \text { for } i=1,2, \ldots, 5 \\
& u_{1}=\frac{A\left(\lambda_{3}-\lambda_{2}\right)}{2 w_{1}}, u_{2}=\frac{S\left(\lambda_{4}-\lambda_{2}\right)}{2 w_{2}}, u_{3}=\frac{Q\left(\lambda_{5}-\lambda_{7}\right)}{2 w_{3}}, u_{4}=\frac{H\left(\lambda_{6}-\lambda_{7}\right)}{2 w_{4}}, u_{5}=\frac{R\left(\lambda_{6}-\lambda_{7}\right)}{2 w_{5}}
\end{aligned}
$$

Moreover, optimal control strategies $u_{1}^{*}, u_{2}^{*}, u_{3}^{*}, u_{4}^{*}, u_{5}^{*}$ are given by:

$$
\begin{aligned}
& u_{1}^{*}=\max \left\{0, \min \left\{1, \frac{A\left(\lambda_{3}-\lambda_{2}\right)}{2 w_{1}}\right\}\right\} \\
& u_{2}^{*}=\max \left\{0, \min \left\{1, \frac{S\left(\lambda_{4}-\lambda_{2}\right)}{2 w_{2}}\right\}\right\} \\
& u_{3}^{*}=\max \left\{0, \min \left\{1, \frac{Q\left(\lambda_{5}-\lambda_{7}\right)}{2 w_{3}}\right\}\right\} \\
& u_{4}^{*}=\max \left\{0, \min \left\{1, \frac{H\left(\lambda_{6}-\lambda_{7}\right)}{2 w_{4}}\right\}\right\} \\
& u_{5}^{*}=\max \left\{0, \min \left\{1, \frac{R\left(\lambda_{6}-\lambda_{7}\right)}{2 w_{5}}\right\}\right\}
\end{aligned}
$$

\section{Numerical simulation}

This section attempts to describe the graphical representation of variations in the model under influence of optimal control strategies. The initialisation of exposed, infected, active and super active spreader, quarantined, hospitalised and recovered is given by $E(0)=10, I(0)=8, A(0)=4, S(0)=4, Q(0)=3, H(0)=4$ and $R(0)=2$ respectively.

Figure 3 shows the variation with time in each compartment of the model. In the initial week of the outbreak, higher intensity of infected, active, and super active spreader is observed, after that, they decrease with time and become negligible in 5-6 weeks. In this duration, since individuals in exposed and hospitalised class are still present, again growth in infection is observed after 7-8 weeks of the outbreak. From this graph, we can say that without any control strategies the infection can reemerge in society after some time of duration. Hence proper control strategies are essential to break the periodic chain of this infection.

Figure $4 \mathrm{a}$ and $\mathrm{b}$ displays the effect of super active spreaders on the class of exposed and infected individuals respectively. Figure $4 \mathrm{a}$ shows that exposed individuals are getting infected by super active spreaders at a high rate. Figure $4 \mathrm{~b}$ shows that infected individuals are moves towards the super active spreaders and 


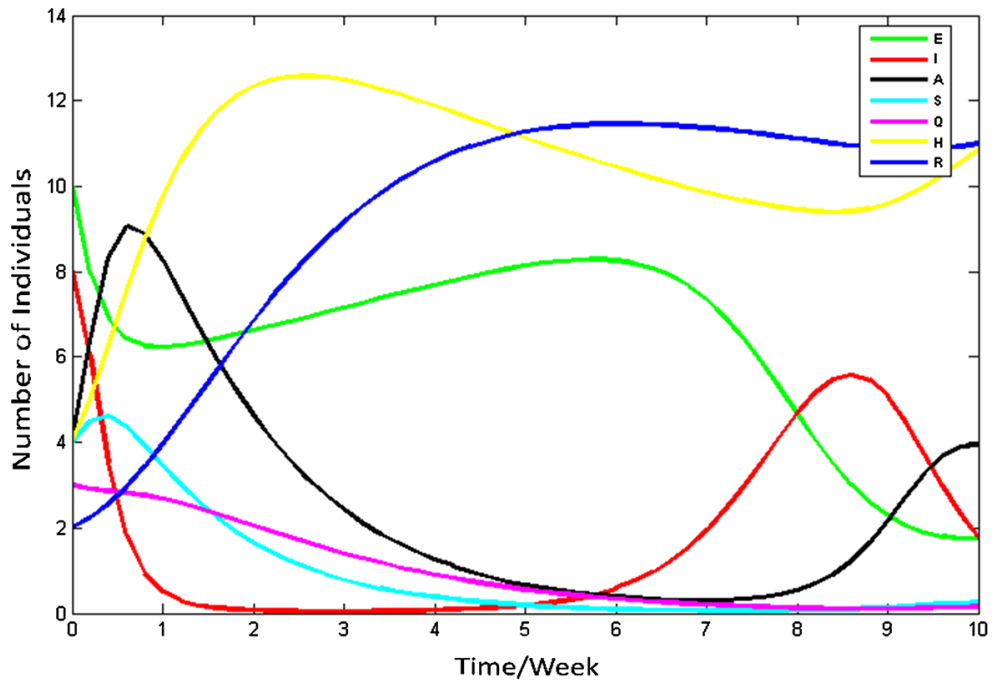

Fig. 3 Variation in compartment with time

they have the tendency to becoming super active spreaders, while super active spreaders are also moving towards the infected class at a lower intensity. This indicates that many super active spreaders getting aware of the disease transmission and they stop spreading the infection by isolation.

Figure 5a shows the intensity of super active spreaders moves towards hospitalisation and Figure $5 \mathrm{~b}$ shows the recovery frequency of super active spreaders.

Periodic transmission of infection through the compartments concerning time (in a week) is observed in Fig. 6. Figure 6a and b show periodic transmission of active and super active spreaders respectively, through hospitalisation and recovery

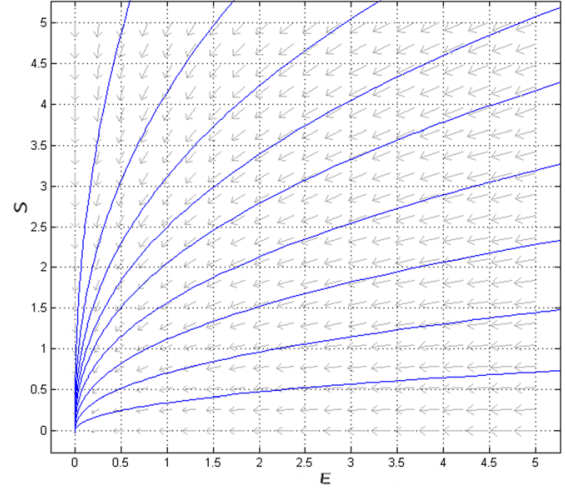

(a)

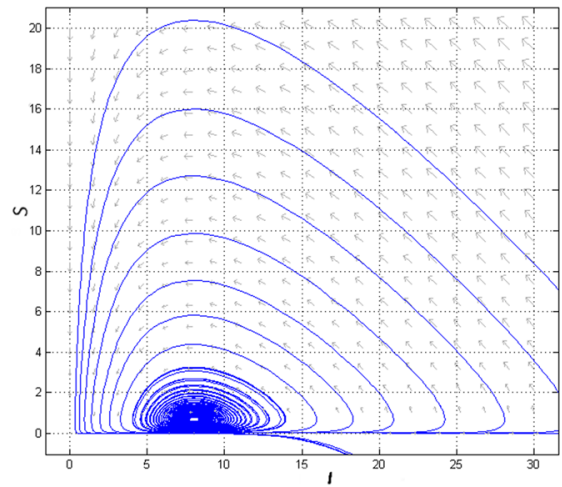

(b)

Fig. 4 Effect of super active spreader on transmission of COVID-19 
class. This scenario suggests that after recovery, still there is a chance of infection. Figure $6 \mathrm{c}$ and $\mathrm{d}$ shows the intensity and periodicity of infected individuals getting recovered by their strong immunity after self-quarantine. Figure 6e shows periodic oscillations between infected, hospitalised and recovered classes. It can be observed from Figure $6 f$ that the infection is moving eriodically around the class of active spreaders, hence we can say that the largest persisting period of the infection is when it is in the class of active spreaders.

Figure 7 shows the oscillations in the model compartments during the outbreak. In the initial days of the outbreak, noteworthy oscillations in the model are observed, moreover, the figure shows that after 100-120 days, the model shows its asymptomatic stability.

\subsection{Susceptibility of $R_{0}$ with respect to the parameters}

To evaluate the intensity of the effect of parameters on the transmission rate of COVID-19, change in the basic reproduction number $\left(R_{0}\right)$ with respect to the most affected parameters of the model is plotted in Fig. 8.

Figure $8 \mathrm{a}$ and $\mathrm{b}$ shows the change in $R_{0}$ concerning to the rate at which infected individuals become active spreaders and super active spreaders respectively. The transmission rate of the infection increases with the rate at which the active spreader becomes a super active spreader. Figure $8 \mathrm{c}$ and d shows change in $R_{0}$ concerning to the rate at which active spreader moves to quarantine cell and hospitalisation respectively. It is observed from the above figures that transmission of the infection is high when rates at which active spreaders are quarantined and hospitalised are low.

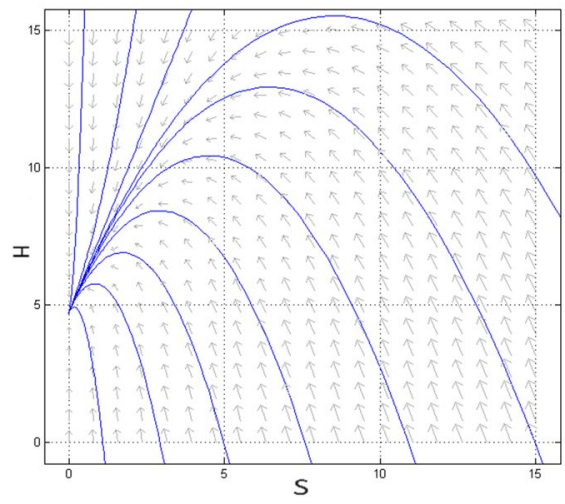

(a)

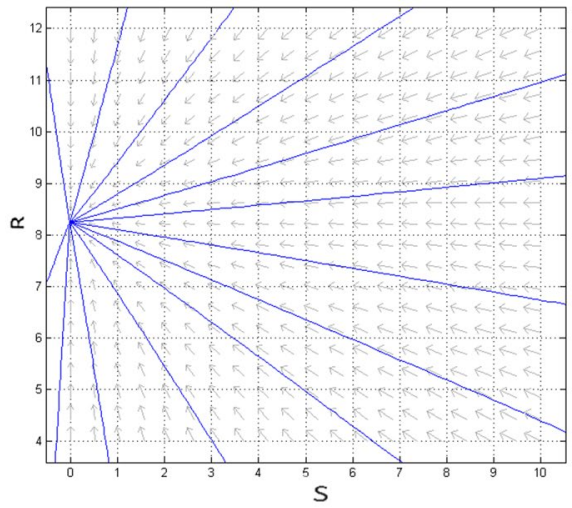

(b)

Fig. 5 Controlling super active spreaders of COVID-19 


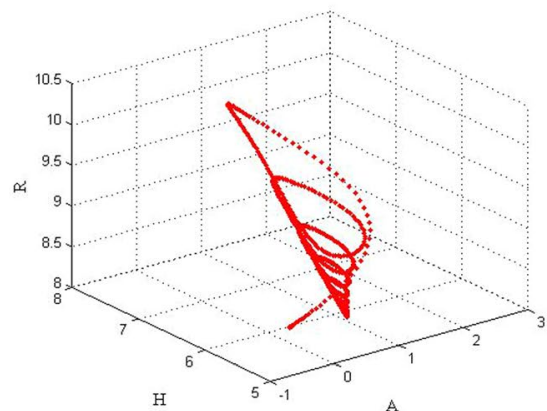

(a)

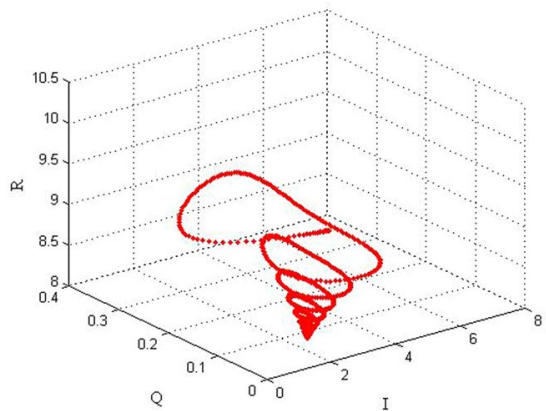

(c)

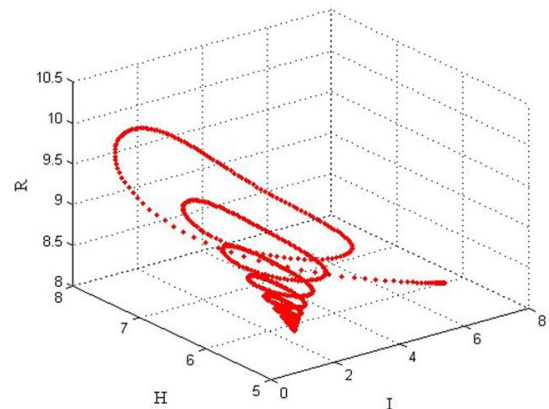

(e)

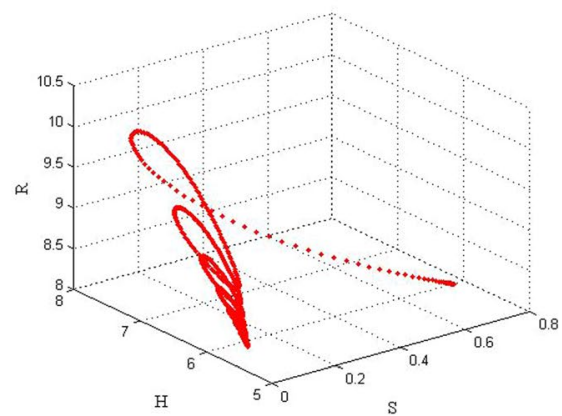

(b)

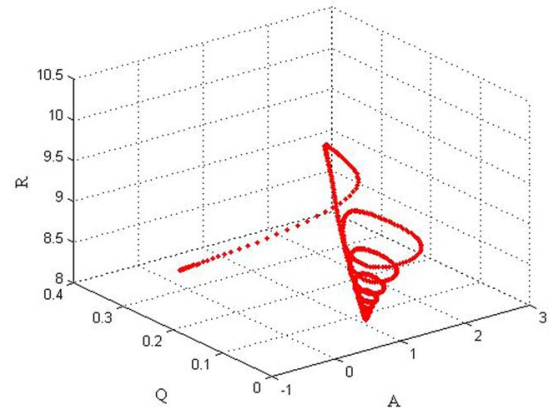

(d)

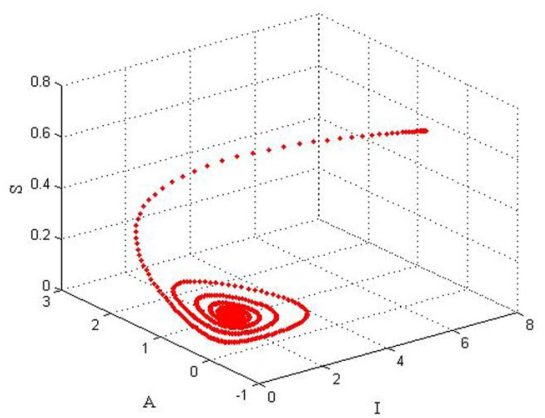

(f)

Fig. 6 Phase portrait diagram of COVID-19 model

\subsection{Simulation influenced by optimal control theory}

In this section, effect of all control strategies on transmission of COVID-19 is observed graphically.

Figure 9 shows the variation in each compartment under influence of with and without control strategies. It is observed that the COVID-19 outbreak can be controlled up to significant level in three weeks after applying all the control strategies together. Super active spreaders are the major threatening problem during this 


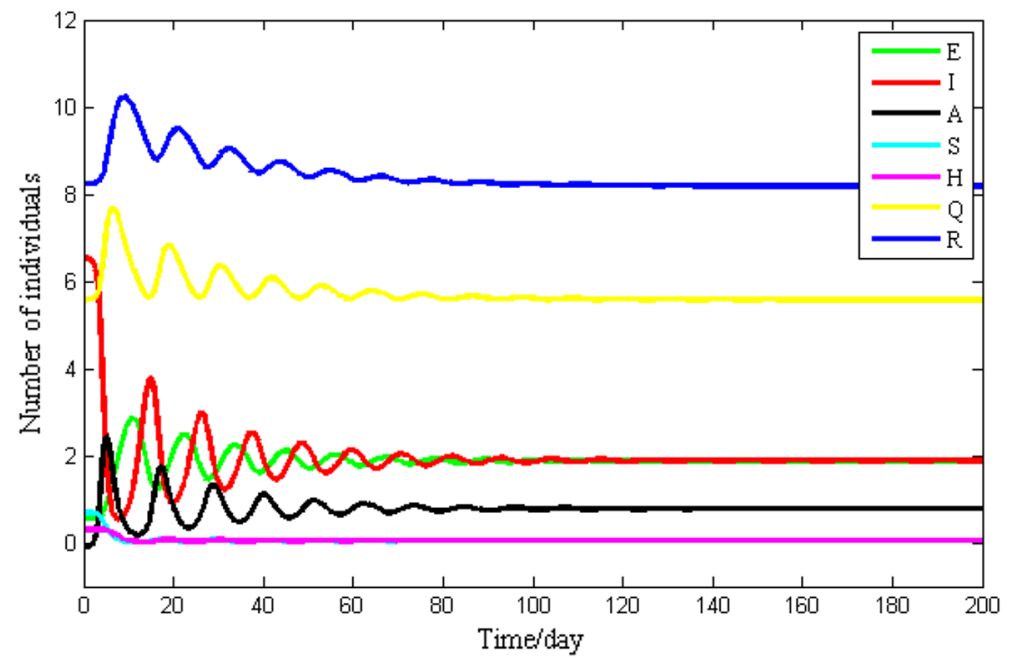

Fig. 7 Oscillations in the model during the COVID-19 outbreak

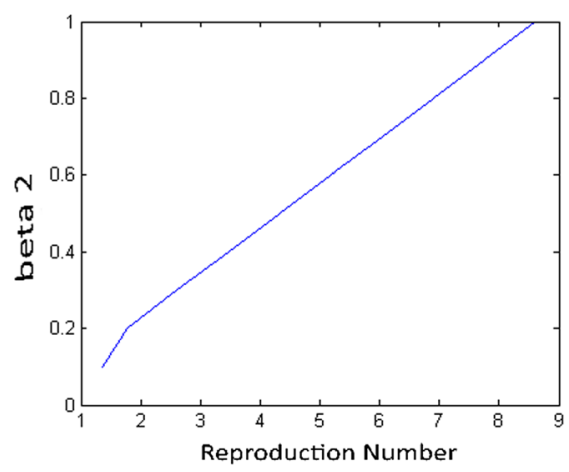

(a)

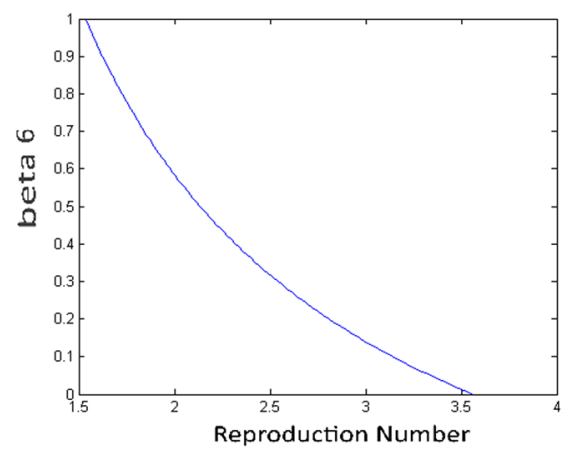

(c)

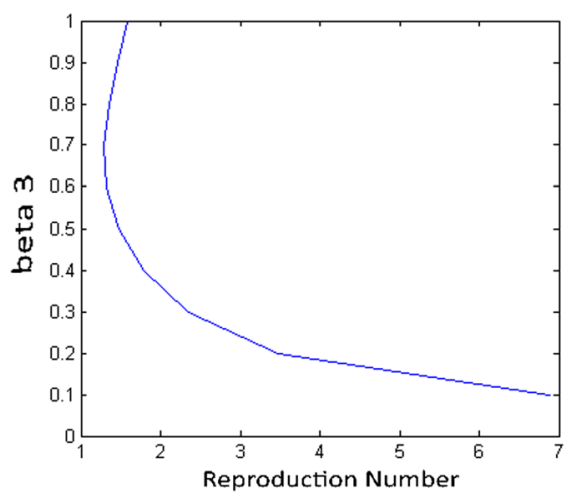

(b)

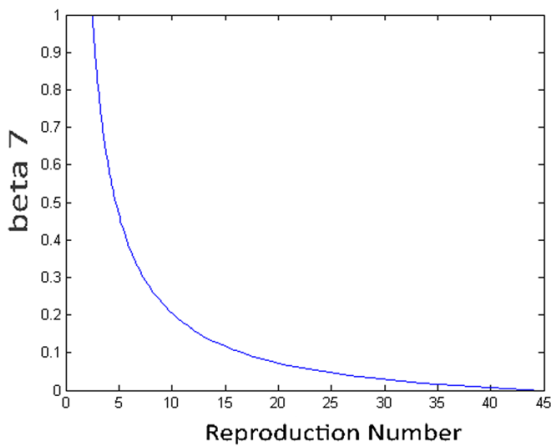

(d)

Fig. 8 Variation in $R_{0}$ with model parameters 
pandemic outbreak. Figure 9d shows that the intensity of the super active spreader is controlled notably after applying the controls which is major factor to reduce the spread of COVID-19. Under this controlled situation reduction in hospitalisation and raise in recovery from the infection is observed in Figure $9 f$ and $g$ respectively.

Figure 10 shows an individual effect of immunotherapy and plasma therapy on the class of recovered individuals. The figure indicates that initially, immunotherapy is highly effective on hospitalised infected individuals, moreover, in long term, better results are observed on recovered class when plasma therapy is applied.

Deviation in the intensity of control strategies with time is shown in Fig. 11. Isolation varies from 10 to $32 \%$, maximum of $21 \%$ quarantine facilities should be used. Using preventive measures, herd immunity can be attained to be $20 \%$. $33 \%$ immune therapy and $10 \%$ plasma therapy should be applied together to fight back the COVID-19 outbreak in around 50 days.

\subsection{Bifurcation analysis}

In the current section, backward bifurcation theory is analysed to understand the behaviour of threshold value of the COVID-19 model. Note that, non-negative equilibria (3) of the COVID-19 model satisfies the quadratic in the infected class $(E)$. Positive equilibrium of the system is achieved by solving the quadratic equation for (z). The bifurcation analysis helps to validate the qualitative information about the basic reproduction number.

The bifurcation diagram is shown in Fig. 12, where blue vertical line indicates the value of the critical point $R_{C}$, which is 2.23 . We can say that this is the point from which system's stability switches from unstable to stable state. To effectively control the spread of COVID-19, the basic reproduction number should be brought below $R_{C}$. Red vertical line in the Fig. 12 shows the numerical value of basic reproduction number. By observing current situation of this pandemic outbreak, it is very hard to bring the value of $R_{0}$ below $R_{C}$ in short time period.

Figure 13 represents the bifurcation diagram of the model with respect to the rate at which infected individuals become super active spreader $\left(\beta_{3}\right)$. Here, the maximum and minimum values of the fluctuations are plotted in blue and red colours respectively. Since the super spreader can create more infection, notable changes in the recovery rate are observed when a gradual change made in the parameter $\beta_{3}$, but it will reduce later.

\section{Discussion and conclusion}

Our study showed that in the initial week of the outbreak, there is a large number of infected people, and behave as active and super active spreaders but in about 5-6 weeks they become negligible. Many super active spreaders are becoming aware of the mode of disease transmission and stop spreading the infection by self-isolation. 


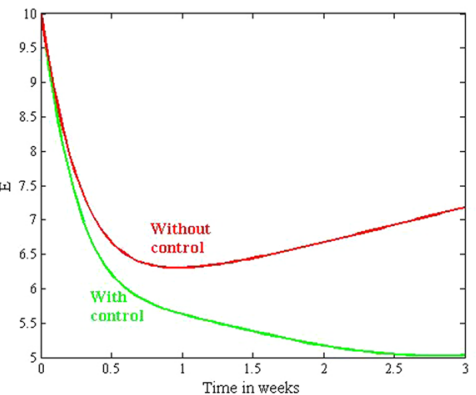

(a)

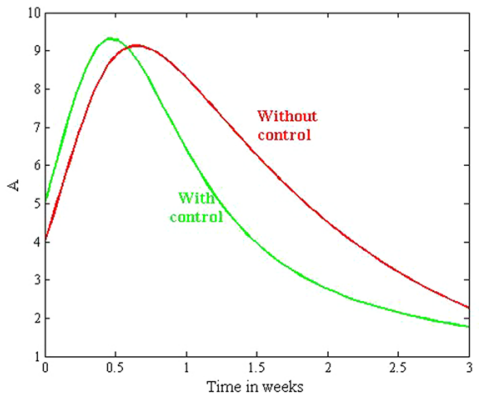

(c)

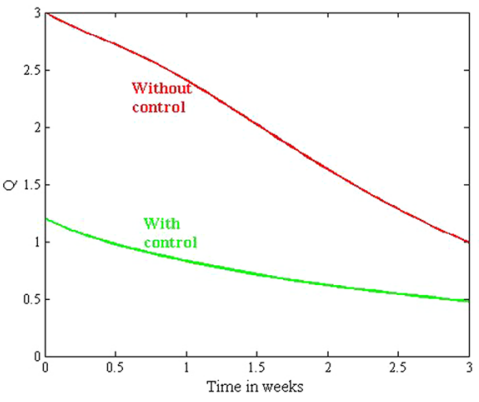

(e)

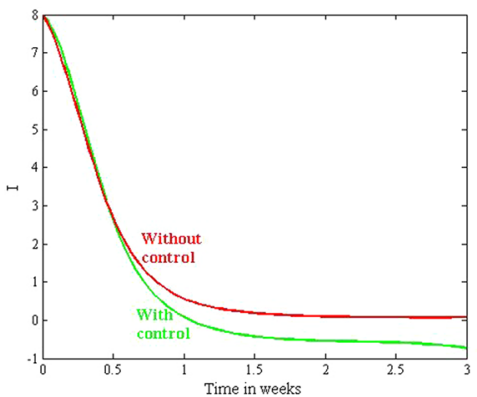

(b)

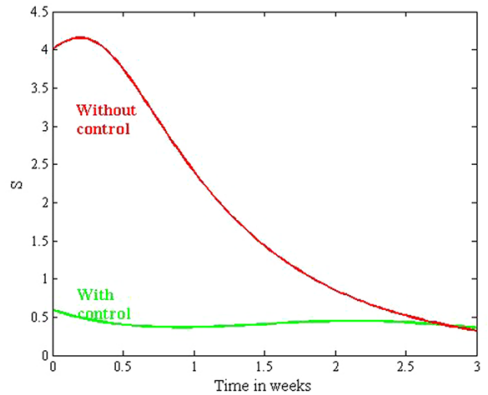

(d)

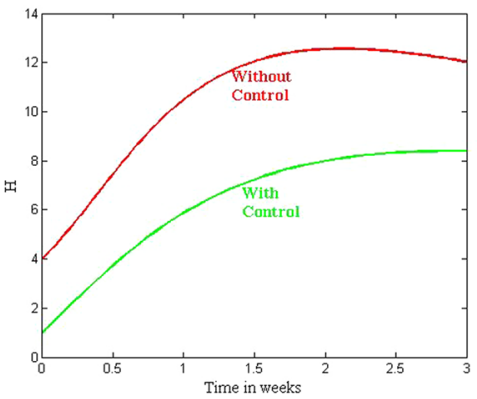

(f)

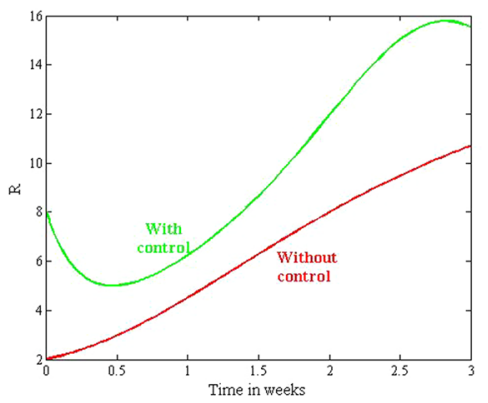

(g)

Fig. 9 Changes in each compartment with and without controls 


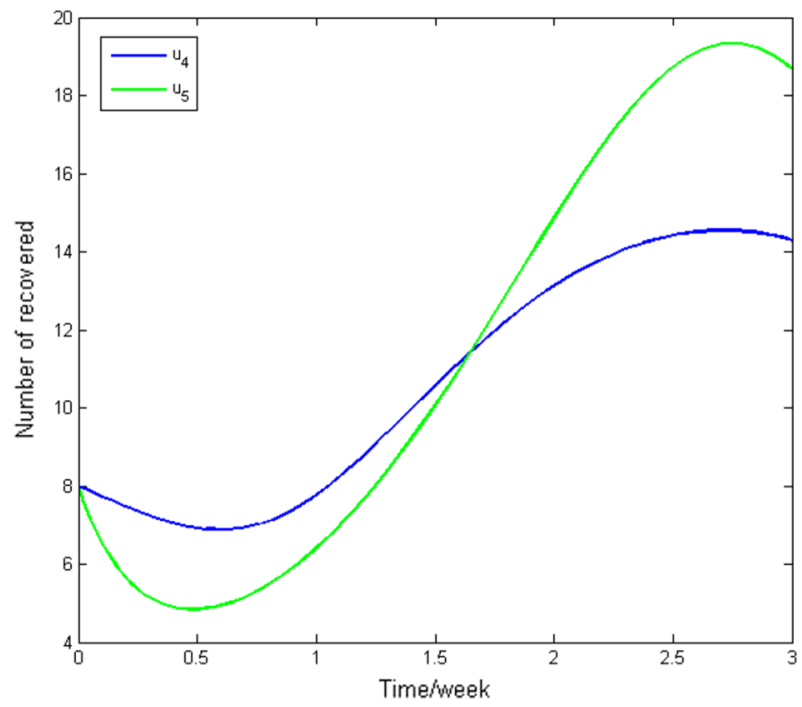

Fig. 10 Effect of immunotherapy and plasma therapy on recovered class

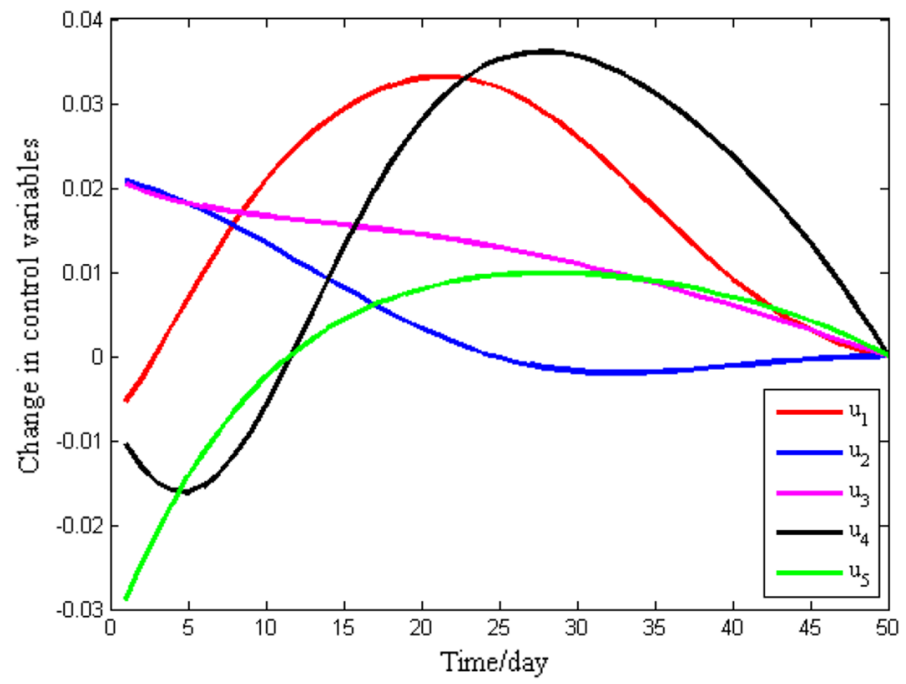

Fig. 11 Change in control variables with time

However, there are still exposed and hospitalized individuals who are responsible for resurgence or second wave of infection at around 7-8 weeks.

Bifurcation analysis showed that $R_{C}$ (critical point) is 2.23 and $R_{0}$ is 3.5 , and by observing current situation of this pandemic, it is very difficult to bring the value of $R_{0}$ below $R_{C}$ in short period of time. All the control measures including selfisolation, quarantine, herd immunity, immunotherapy and plasma therapy should be 


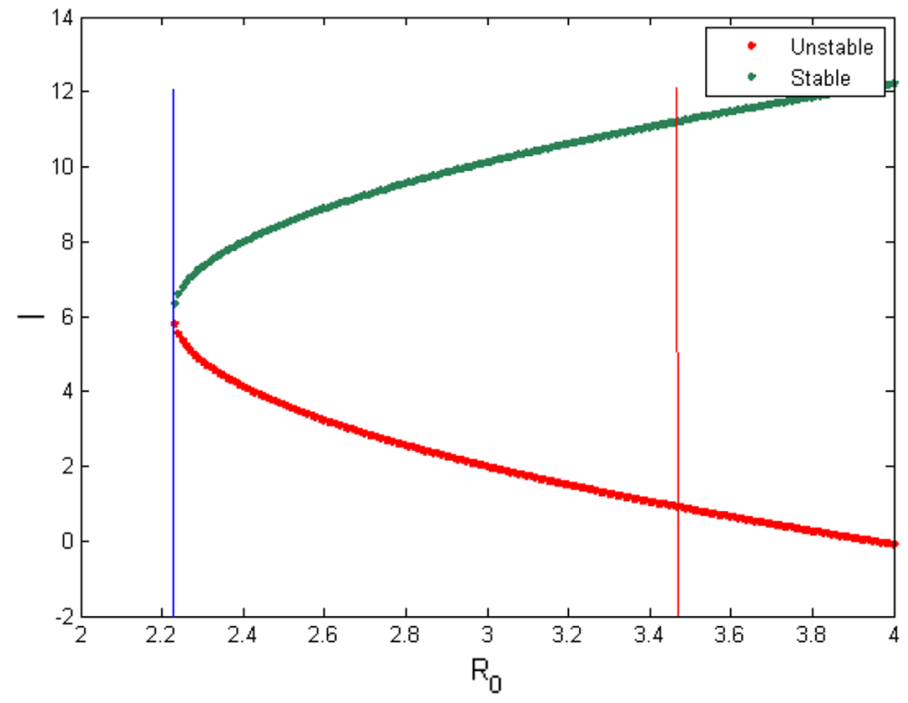

Fig. 12 Bifurcation diagram for the COVID-19 model

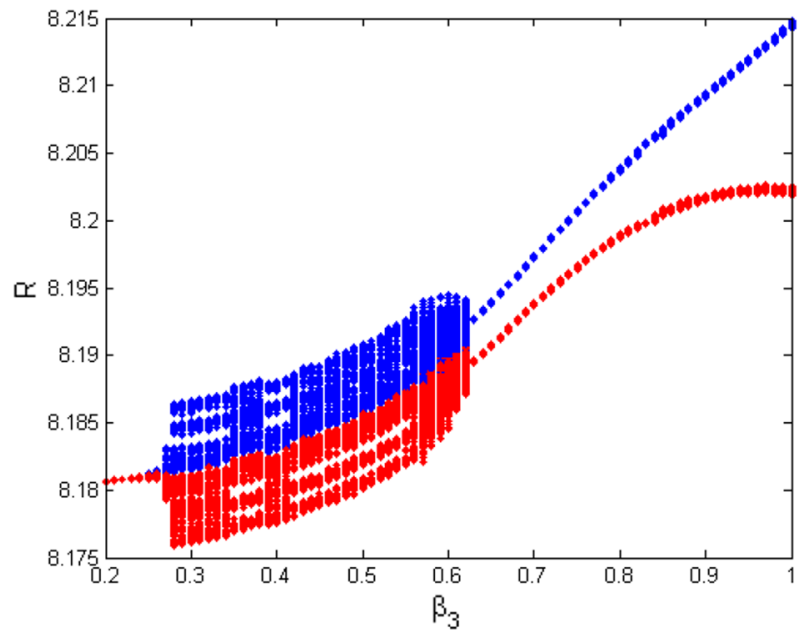

Fig. 13 Bifurcation w.r.t $\beta_{3}$

applied together to fight back COVID-19 in around 50 days. Effectiveness of selfisolation varies between 10 and $32 \%$, quarantine is about $21 \%$, herd immunity is about $20 \%$, immune therapy is $33 \%$ and plasma therapy is about $10 \%$. The optimal timing of the plasma therapy is around 15 days from the infection while immunotherapy should be implemented earlier to get maximum benefit.

Thus, we conclude that the COVID-19 outbreak can be controlled up to a significant level in three weeks after applying all the control strategies including 
self- isolation, quarantine and hospitalization together. Furthermore, proper control strategies are of paramount importance in breaking this periodic chain of infection and preventing resurgence of infection. Super active spreaders are the major threatening problem during this pandemic outbreak. Our results show that the super active spreaders can be controlled notably by optimal controls strategies. These strategies lead to a reduction in hospitalization and rise in recovery from infection. Immunotherapy is highly effective initially in hospitalized infected individuals however better results were seen in the long term with plasma therapy.

We also suggest certain policies to make sure that plasma therapy is available on a larger scale. Physician can motivate patients at the time of discharge to donate plasma in near future (once they are eligible to donate). Furthermore, the government should expand plasma collection capabilities. They need to create infrastructure and remove certain barriers for agencies to prioritize collecting plasma and making it available on a larger scale for treatment at subsequent waves of COVID-19 infection.

Preliminary data using immunotherapy and plasma therapy against the rapidly increasing number of COVID-19 cases provides an unprecedented opportunity to perform large-scale randomized clinical trials, to study the efficacy of this treatment against a viral agent. If the results of rigorously conducted, investigations demonstrate consistent efficacy, use of these therapies could help change the course of this pandemic. The suggestions made here are dependent on model parameters and suspect to vary depending on the intensity of disease spread and geographical area of the study.

Acknowledgements All the authors are thankful to reviewers for their constructive comments. All the authors are thankful to DST-FIST file \# MSI-097 for technical support to the Department of Mathematics, Gujarat University. Second author (AHS) is funded by a Junior Research Fellowship from the Council of Scientific \& Industrial Research (file no.-09/070(0061)/2019-EMR-I). Third author (ENJ) is funded by UGC granted National Fellowship for Other Backward Classes (NFO-2018-19-OBC-GUJ-71790).

\section{Declarations}

Conflict of interest The authors declare that we have no known competing financial interests or personal relationships that could have appeared to influence the work reported in this paper.

\section{References}

AminJafari A, Ghasemi S. The possible of immunotherapy for COVID-19: a systematic review. Int Immunopharmacol. 83, 106455. (2020). https://doi.org/10.1016/j.intimp.2020.106455

Bloch, E.M., Shoham, S., Casadevall, A., Sachais, B.S., Shaz, B., Winters, J.L., et al.: Deployment of convalescent plasma for the prevention and treatment of COVID-19. J. Clin. Invest. 130(6), 27572765 (2020)

Chen, L., Xiong, J., Bao, L., Shi, Y.: Convalescent plasma as a potential therapy for COVID-19. Lancet Infect. Dis. 20(4), 398-400 (2020)

Cheng, Y., Wong, R., Soo, Y.O.Y., Wong, W.S., Lee, C.K., Ng, M.H.L., et al.: Use of convalescent plasma therapy in SARS patients in Hong Kong. Eur. J. Clin. Microbiol. Infect. Dis. 24(1), 44-46 (2005)

Cohen, J., Normile, D.: New SARS-like virus in China triggers alarm. Science 367, 234-235 (2020) 
Diekmann, O., Heesterbeek, J.A.P., Metz, J.A.J.: On the definition and the computation of the basic reproduction ratio R0 in models for infectious diseases in heterogeneous populations. J. Math. Biol. (1990). https://doi.org/10.1007/BF00178324

Duan, K., Liu, B., Li, C., Zhang, H., Yu, T., Qu, J., et al.: Effectiveness of convalescent plasma therapy in severe COVID-19 patients. Proc. Natl. Acad. Sci. 117(17), 9490-9496 (2020)

Duan K, Liu B, Li C, et al. The feasibility of convalescent plasma therapy in severe COVID-19 patients: a pilot study. medRxiv; 2020. https://doi.org/10.1101/2020.03.16.20036145.

Fleming, W., Rishel, R.: Deterministic and stochastic optimal control. deterministic and stochastic optimal control. Springer, Newyork (1975)

Garba, S.M., Gumel, A.B., Abu Bakar, M.R.: Backward bifurcations in dengue transmission dynamics. Math. Biosci. 215(1), 11-25 (2008)

Holshue, M.L., DeBolt, C., Lindquist, S., Lofy, K.H., Wiesman, J., Bruce, H., et al.: First case of 2019 novel coronavirus in the United States. N Engl. J. Med. 382(10), 926-936 (2020)

Hung, I.F.N., To, K.K.W., Lee, C.K., Lee, K.L., Chan, K., Yan, W.W., et al.: Convalescent plasma treatment reduced mortality in patients with severe pandemic influenza A (H1N1) 2009 virus infection. Clin. Infect. Dis. 52(4), 447-456 (2011)

Ko, J.H., Seok, H., Cho, S.Y., Ha, Y.E., Baek, J.Y., Kim, S.H., et al.: Challenges of convalescent plasma infusion therapy in Middle East respiratory coronavirus infection: a single centre experience. Antivir. Ther. 26(7), 617-622 (2018)

Lee, P.I., Hsueh, P.R.: Emerging threats from zoonotic coronaviruses-from SARS and MERS to 2019nCoV. J. Microbiol. Immunol. Infect. 53(3), 365-367 (2020)

Lu H. (2020) Drug treatment options for the 2019-new coronavirus (2019-nCoV). Biosci Trends

Mahrouf, M., et al.: Modeling and forecasting of COVID-19 spreading by delayed stochastic differential equations. Axioms 10 (2021). https://doi.org/10.3390/axioms10010018

Mair-Jenkins, J., Saavedra-Campos, M., Baillie, J.K., Cleary, P., Khaw, F.M., Lim, W.S., et al.: The effectiveness of convalescent plasma and hyperimmune immunoglobulin for the treatment of severe acute respiratory infections of viral etiology: a systematic review and exploratory meta-analysis. $\mathbf{J}$ Infect Dis. 211(1), 80-90 (2015)

Ndaïrou, F., Area, I., Nieto, J.J., Torres, D.F.: Mathematical modeling of COVID-19 transmission dynamics with a case study of Wuhan. Chaos, Solitons \& Fractals 135, 109846 (2020)

Pontryagin LS. Mathematical theory of optimal processes. Routledge; 2018 May 3

Russell, C.D., Millar, J.E., Baillie, J.K.: Clinical evidence does not support corticosteroid treatment for 2019-nCoV lung injury. Lancet 395, 473-475 (2020)

Shen C, Wang Z, Zhao F, et al. Treatment of 5 Critically Ill Patients With COVID-19 With Convalescent Plasma. JAMA. 323(16), 1582-1589. (2020). https://doi.org/10.1001/jama.2020.4783

Syal, K.: COVID-19: Herd immunity and convalescent plasma transfer therapy. J. Med. Virol. 92(9), 1380-1382 (2020)

Van den Driessche, P., Watmough, J.: Reproduction numbers and sub-threshold endemic equilibria for compartmental models of disease transmission. Math. Biosci. 180(1-2), 29-48 (2002)

Wang, M., Cao, R., Zhang, L., Yang, X., Liu, J., Xu, M., et al.: Remdesivir and chloroquine effectively inhibit the recently emerged novel coronavirus (2019-nCoV) in vitro. Cell Res. 30(3), 269-271 (2020)

Worldometer. (2020) Coronavirus Update (Live): 472,907 Cases and 21,315 Deaths from COVID-19 Virus Outbreak - Worldometer. Worldometer

Zhou, B., Zhong, N., Guan, Y.: Treatment with convalescent plasma for influenza A (H5N1) infection. New Engl. J. Med. 357(14), 1450-1451 (2007)

Publisher's Note Springer Nature remains neutral with regard to jurisdictional claims in published maps and institutional affiliations. 


\section{Authors and Affiliations}

Nita H. Shah ${ }^{1} \cdot$ Ankush H. Suthar ${ }^{1}$ - Ekta N. Jayswal ${ }^{1} \cdot$ Nehal Shukla $^{2}$ (D) Jagdish Shukla ${ }^{3}$

Nita H. Shah

nitahshah@gmail.com

Ankush H. Suthar

ankush.suthar1070@gmail.com

Ekta N. Jayswal

jayswal.ekta1993@gmail.com

Jagdish Shukla

jagdish.shukla@piedmont.org

1 Department of Mathematics, Gujarat University, Ahmedabad, Gujarat 380009, India

2 Department of Mathematics, Columbus State University, Columbus, Georgia 31907, USA

3 Department of Medical Education, Piedmont Columbus Regional Health Care, Columbus, Georgia 31901, USA 